\title{
ACESSO AO SERVIÇO DE FONOAUDIOLOGIA: A IMPLANTAÇÃO DO ACOLHIMENTO NO MUNICÍPIO DE TOLEDO - PR
}

\section{Access to service of speech therapy: implementing the embracement practice in Toledo City - PR}

\author{
Juliana Cunha da Costa ${ }^{(1)}$, Schirley Aparecida Giustti (2), leda Satiko Murofuse ${ }^{(3)}$, Anely Lea Gumz (4)
}

\begin{abstract}
RESUMO
Tema: para inserir o acolhimento nos serviços de saúde, é preciso desenvolver formas de receber a população; respeitando os diferentes modos de como o usuário procura ajuda. Desta forma, o presente trabalho é um relato de experiência, que teve por objetivo agilizar o acesso ao serviço de Fonoaudiologia, bem com escutar e dar respostas mais adequadas aos usuários. Procedimentos: como forma de estruturar o acesso foi realizada a implantação das práticas de acolhimento na porta de entrada ao serviço de Fonoaudiologia do município de Toledo, no Paraná. Para a realização do acolhimento foram estabelecidos dois momentos. O primeiro referiu-se a lista de espera de usuários que necessitava ser conhecida e no momento posterior, os novos usuários escolheram entre dias e horários fixos, como forma de entrada no serviço de Fonoaudiologia. Em alguns encontros houve também a participação de enfermeiro, psicólogo e assistente social. Resultados: ofereceu-se aos usuários melhor acesso ao atendimento fonoaudiológico, proporcionou o enriquecimento da relação profissional/usuário, à adequação dos procedimentos terapêuticos e esclarecimentos quanto ao funcionamento do serviço. Conclusão: a implantação do acolhimento na porta de entrada ao serviço de Fonoaudiologia agilizou o acesso da população, assim como escutar implica em respeitar as demandas do usuário.
\end{abstract}

DESCRITORES: Acolhimento; Acesso aos Serviços de Saúde; Fonoaudiologia

\section{INTRODUÇÃO}

Atualmente, tem-se discutido a implantação do acolhimento nas práticas de saúde como diretriz ética/estética/política constitutiva dos modos de se produzir saúde e ferramenta tecnológica de intervenção na qualificação de escuta, construção de

(1) Fonoaudióloga; servidora pública da Prefeitura Municipal de Toledo, Toledo, PR, Brasil; Especialização em Saúde Pública pela Universidade Estadual do Oeste do Paraná e Especialização em Fonoaudiologia pela Universidade Federal de Santa Maria.

(2) Fonoaudióloga; servidora pública da Prefeitura Municipal de Toledo, Toledo, PR, Brasil.

(3) Fonoaudióloga; servidora pública da Prefeitura Municipal de Toledo, Toledo, PR, Brasil.

(4) Fonoaudióloga; servidora pública da Prefeitura Municipal de Toledo, Toledo, PR, Brasil.

Conflito de interesses: inexistente vínculo, garantia do acesso com responsabilização e resolutividade nos serviços ${ }^{1}$. Reconhece-se que para o acolhimento se tornar efetivo é necessário repensar o processo de trabalho em saúde sendo, portanto, um desafio para os profissionais da área.

A implementação do acolhimento como ferramenta em uma das etapas do processo de trabalho em saúde, surgiu a partir de uma necessidade do setor de Fonoaudiologia em reestruturar as formas de atendimento aos usuários do Sistema Único de Saúde (SUS). Após o conhecimento de práticas de acolhimento realizadas em outros estados brasileiros, iniciaram-se os estudos sobre este tema, utilizando-se artigos científicos e os textos básicos de saúde elaborados pelo Ministério da Saúde sobre a Política Nacional de Humanização do SUS (Humaniza SUS). Entende-se por humanização a valorização dos diferentes sujeitos implicados no 
processo de produção de saúde. Os valores que norteiam essa política são a autonomia e o protagonismo dos sujeitos, a co-responsabilidade entre eles, os vínculos solidários e a participação coletiva no processo de gestão².

É relevante lembrar que foi necessária uma iniciativa das próprias fonoaudiólogas para que estas políticas pudessem ser implantadas, uma vez que estes materiais com informações atualizadas raramente chegam aos profissionais de saúde por ação da gestão.

Para contextualizar o acolhimento, é importante referir que as tecnologias no trabalho em saúde podem ser classificadas como leve, levedura e dura ${ }^{3}$. Sendo a tecnologia leve a relação de produção de vínculos, acolhimento, autonomia e gestão como um modo de governar processos de trabalho, que são fundamentais para a produção do cuidado e referem-se à atitude própria do profissional que é influenciado por uma intencionalidade aliada ao campo cuidador e à sua subjetividade. A tecnologia leve-dura caracteriza-se por uma parte leve, que é o modo próprio como cada trabalhador a utiliza, podendo assumir diversas formas, dependendo sempre da maneira como cada um trabalha e cuida do usuário, e por outra parte dura, que é o conhecimento técnico e saberes bem estruturados que agem na saúde, como a epidemiologia, a clínica médica e psicanalítica. Já, a tecnologia dura refere-se aos equipamentos tecnológicos como as máquinas e normas.

Como forma de tecnologia leve, o acolhimento, tem por objetivo atender a todos que procuram os serviços de saúde, assumindo no serviço uma postura capaz de acolher, escutar e dar respostas mais adequadas aos usuários, ou seja, atuar com resolutividade e responsabilização assistindo, orientando e encaminhando de acordo com as necessidades de cada caso ${ }^{1}$.

A implementação do acolhimento exige mudança de postura em todo o sistema de saúde, a fim de receber os casos e de responsabilizar-se de modo integral por eles. Para acolher é preciso desenvolver formas de receber a população, de acordo com os diferentes modos como ela procura ajuda nos serviços de saúde, sem impor limites e respeitando o momento existencial de cada $\mathrm{um}^{4}$. Acolher não é simples, e demanda energia. Com o acolhimento, as pessoas passam a ser ouvidas em espaço físico mais reservado e têm a oportunidade de falar de seus problemas com um profissional ${ }^{5}$, tendo assim, suas demandas melhor atendidas. $\mathrm{O}$ acolhimento é uma possibilidade de mudança do processo de trabalho a fim de atender a todos que procuram o serviço de saúde ${ }^{6}$.
Anteriormente a implantação do acolhimento, o setor de Fonoaudiologia era organizado através da realização de triagens individuais, as quais incluíam anamnese, avaliação e orientação fonoaudiológica, onde também eram feitos os encaminhamentos pertinentes. Com essas triagens, o serviço possuía uma lista de espera de usuários que não haviam tido uma escuta e que, por este motivo, não se conheciam as reais demandas, tais como a necessidade de fonoterapia, de encaminhamentos para exames complementares e/ou outras especialidades. Assim, o acolhimento surgiu como um facilitador da problemática da porta de entrada do serviço e da conduta dada aos usuários.

Por existirem grandes lacunas nos modelos de atenção e gestão dos serviços no que se refere ao acesso e ao modo como o usuário é acolhido nos serviços de saúde pública ${ }^{1}$, o objetivo do presente trabalho foi relatar as ações desenvolvidas para agilizar o acesso ao serviço de Fonoaudiologia na cidade de Toledo -PR.

\section{APRESENTAÇÃO DO CASO}

O serviço de Fonoaudiologia funciona no Centro de Atendimento à Saúde e conta também com profissionais das áreas de Psicologia, Terapia Ocupacional, Serviço Social, Psicopedagogia, Neuropediatria, Enfermagem e Psiquiatria, o que possibilita um trabalho interdisciplinar. Porém, sabe-se que para uma mudança no processo de trabalho de um serviço de saúde acontecer são necessárias modificações nas concepções advindas da formação acadêmica; de flexibilidade para novos modos de fazer e do interesse em realizar atividade interdisciplinar, entre outros. Assim, por estes fatores e como forma de experimentar uma nova proposta para posteriormente sugeri-la aos demais profissionais, iniciou-se o acolhimento por área, que neste serviço iniciou-se pela Fonoaudiologia.

No município de Toledo-PR, o usuário pode ter acesso ao serviço de Fonoaudiologia por iniciativa própria, entretanto, uma significante parte dos usuários chega ao serviço por meio de encaminhamentos de escolas e outros profissionais de saúde. A população é informada sobre o acolhimento em Fonoaudiologia por meio de cartazes afixados nas Unidades Básicas de Saúde e nas escolas municipais.

É importante referir que se optou por acolhimentos grupais uma vez que é necessário investir na preservação da riqueza concreta dos objetos e das experiências institucionais, especialmente no que se refere a pensar nas práticas grupais como escolha ética, estética e política, acreditando na construção de uma outra produção subjetiva: os 
coletivos como estratégia de quebrar formas solitárias de viver ${ }^{7}$. Grupalidade é um coletivo ou uma multiplicidade de termos (usuários, trabalhadores, gestores, familiares, etc.) em agenciamento e transformação, compondo uma rede de conexão na qual o processo de produção de saúde e de subjetividade se realiza ${ }^{8}$.

Para a realização do acolhimento foram estabelecidos dois momentos. O primeiro, referiu-se a lista de espera de usuários que necessitava ser conhecida, desta forma, respeitando a ordem desta, foram efetuados com êxito, 136 contatos telefônicos com os usuários da referida lista para esclarecimento da possível queixa, e estes foram agrupados por patologias (desvio fonético e/ou fonológico, distúrbio de leitura e escrita, gagueira, disfonia) e agendados para participarem do acolhimento, divididos ao longo de quinze encontros. Já era informado por contato telefônico que o não comparecimento sem justificativa plausível ao acolhimento ocasionaria a perda da vaga. Com isso, observou-se uma significante redução na lista de espera.

Estes acolhimentos por patologia tiveram a duração de cinco meses para que pudesse ser atendida toda a demanda da lista de espera. E esta forma possibilitou a realização de orientações específicas e os encaminhamentos necessários.

Após o acolhimento destes pacientes, os novos usuários puderam escolher entre dias e horários fixos, pré-determinados, por semana, como forma de entrada no serviço de Fonoaudiologia. Sabe-se que o acolhimento é uma postura ética e não é um espaço ou um local, nem pressupõe hora ou profissional específico para fazê-lo ${ }^{1}$, porém, pelas atividades que envolvem o atendimento fonoaudiológico no Centro de Atendimento à Saúde foi necessário estabelecer horários para a entrada de novos usuários.

Primeiramente, atenderam-se apenas alguns aspectos da coletividade, contudo almejava-se contemplar o indivíduo como um todo. A partir deste desejo, colocou-se à equipe multiprofissional o convite para que o profissional interessado participasse dos acolhimentos. Com este convite, obteve-se a adesão da assistente social e, posteriormente, da enfermeira e dos psicólogos. Deste modo, os acolhimentos foram enriquecidos pelos diferentes olhares em relação ao indivíduo, sendo que os profissionais ainda passaram a contribuir no acolhimento.

Esta interdisciplinaridade foi essencial, uma vez que nos acolhimentos muitas das queixas não permeavam apenas aspectos fonoaudiológicos, pois eram observados relatos como dificuldades motoras, agitação, nervosismo, condições socioeconômicas precárias, dores e sensações específicas, entre outros aspectos, que necessitavam de apreciações distintas.

Nos momentos em que se mostravam possíveis também eram realizadas orientações, ao mesmo tempo em que as profissionais ficavam a disposição para sanar possíveis dúvidas.

\section{RESULTADOS}

Como resultados, ofereceu-se aos usuários uma acessibilidade ao atendimento fonoaudiológico um pouco mais coerente com os propósitos do SUS. Do mesmo modo possibilitou-se a significativa relação de confiança e identificação entre profissionais/usuários, estabelecendo vínculos entre os mesmos. Facilitou-se também a informação quanto ao funcionamento do serviço e esclarecimentos sobre o próprio fazer da Fonoaudiologia, ao mesmo tempo em que deu mais agilidade e precisão quanto aos procedimentos terapêuticos.

Durante os acolhimentos priorizou-se a escuta do usuário quanto a aspectos que o mesmo queira relatar, retirando-se 0 enfoque do que o profissional quer ouvir. E nestes momentos, foi possível observar o real desejo dos usuários para o tratamento, a partir de perguntas como "O que você espera do tratamento?", "Você está disposto a nos ajudar?", assim como, verificar se o motivo da procura pelo atendimento refere-se a uma demanda da escola ou do usuário e/ou família. Quando foi possível, realizaram-se orientações aos usuários e/ou familiares e o encaminhamento de acordo com as necessidades de cada caso, respeitando a demanda do usuário.

Aconteceram ainda, nessas ocasiões, discussões quanto a questões como o processo de inclusão, a discriminação, o uso de medicação, as dificuldades escolares, entre outros, sendo que esses assuntos partiam dos próprios usuários.

$\mathrm{O}$ acolhimento em grupo apresentou-se como um valioso modo de realizar o trabalho em saúde, pois desta forma houve a identificação do usuário com o outro, a qual o indivíduo ou familiar comparava as dificuldades, da mesma forma que alguns usuários, à procura do atendimento, verificavam que seus filhos ou o próprio responsável também apresentavam necessidade de atendimento fonoaudiológico. Como também, existiram casos que não precisavam da terapia fonoaudiológica propriamente dita.

A partir do acolhimento realizado pela Fonoaudiologia, constatou-se que os demais profissionais da equipe multiprofissional também tiveram o interesse em conhecer e estudar a Política Nacional do Humaniza SUS. 


\section{DISCUSSÃO}

O acolhimento e o acesso são inseparáveis, pois a acessibilidade é entendida como um dos aspectos da oferta do serviço em relação à capacidade de responder às demandas. Sendo assim, não é apenas a oferta de serviços de saúde, mas as características do serviço e dos recursos de saúde que facilitam ou limitam seu uso por potenciais usuários. Em seu aspecto organizacional, remete ao desenho do serviço, e o acesso está diretamente atrelado ao acolhimento, que se refere ao primeiro contato com quem nos procura, a partir do qual será definida toda a sequência de atendimentos. É o momento em que todos os recursos do serviço são postos à prova: quem atende; o que pode oferecer; como pode acolher, avaliar e discriminar a demanda ${ }^{9}$. Portanto, a partir do primeiro contato do usuário com o serviço, o mesmo pode conhecer as fonoaudiólogas, o local e as possibilidades de atendimento, sabendo o que e quem procurar dentro do serviço. Estes aspectos foram observados nas posteriores buscas do usuário junto ao Centro de Atendimento à Saúde.

Assim sendo, o acolhimento vai além do oferecido pelo profissional que presta o atendimento, permeando os demais encontros que ocorrem durante a permanência do usuário no serviço de saúde, desde sua recepção até seu atendimento propriamente dito. Como define o Humaniza SUS, é necessário não restringir o acolhimento apenas ao problema da recepção da demanda espontânea, tratando-o como algo que qualifica uma relação e é, portanto, passível de ser apreendido e trabalhado em todo e qualquer encontro e não apenas numa condição particular como é o caso da recepção. $O$ acolhimento na porta de entrada só ganha sentido se $o$ entendermos como uma passagem para 0 acolhimento nos processos de produção de saúde, dentre eles, a terapia ${ }^{10}$.

Corroborando com estas informações, verificouse que o acolhimento possibilitou a formação de vínculos entre profissionais e usuários, e considerando este aspecto, o diálogo horizontal com os usuários e a relevância dada às suas opiniões e desejos trazem ao serviço um perfil de espaço terapêutico e cuidado integral à saúde, proporcionando, também, maior aproximação do profissional ao modo de viver e sentir as necessidades trazidas ao espaço pela população ${ }^{11}$. Deste modo, os trabaIhadores e o serviço como um todo, devem estabelecer uma relação humanizada e acolhedora com os diferentes tipos de usuários que a eles procuram ${ }^{12}$.

Autores também destacaram que para realizar um melhor acolhimento e garantir o acesso são necessárias ações mais diversificadas e pouco ritualizadas com profissionais mais flexíveis e versáteis, sendo que quanto mais as pessoas estão trabalhando juntas, mais aberto e acessível o serviço está a todos os tipos de demanda, aumentando a possibilidade de a equipe imergir no mundo sociocultural de sua área de abrangência, de trocar saberes pessoais e profissionais ${ }^{13}$. Com a inserção de psicólogos, assistente social e enfermeira no acolhimento da área de fonoaudiologia, foi possível um trabalho interdisciplinar, e sabendo que o processo de trabalho na saúde é coletivo e, por conseguinte, cada grupo de ações, realizado por diferentes categorias profissionais, é parte do processo de trabalho que é presidido por uma finalidade única e maior, que compreende todos os trabalhos aí contidos, não há como indicar determinadas funções para as diferentes categorias, sem que previamente tenha se decidido a qual finalidade o trabalho na saúde vai, quer ou pode atender ${ }^{14}$. Assim, trabalhando em equipe, a Psicologia, Assistência Social e Enfermagem contribuíram especialmente em queixas como falta de limites em crianças, retirada de sucção digital e hábitos orais deletérios, condições de vida precárias e demais hábitos insuficientes de higiene.

O acolhimento priorizou a escuta do usuário quanto a aspectos que o mesmo queira relatar, retirando-se o enfoque do que o profissional quer ouvir. Sendo que a literatura refere que existem duas possibilidades de entendimento do acolhimento: uma como postura diante do usuário e suas necessidades, de contínua investigação e negociação das necessidades de saúde e modos de satisfazê-las em todos os momentos do processo de produção de serviços de saúde. E outra, como dispositivo capaz de reorganizar o trabalho na unidade, uma etapa do processo de trabalho que tem como objetivo atender à demanda espontânea, aumentando o acesso e humanizando as ações receptoras dos usuários no serviço. Citam ainda, que quando o serviço possui um canal de escuta com o usuário, abre uma possibilidade para que as pessoas tragam, para dentro do serviço de saúde, as suas queixas, problemas e necessidades. Ouvir não é tarefa fácil. É mais simples trabalhar com uma "queixa clínica", uma parte do corpo que dói, uma doença que precisa de medicamento. Quando abrimos um espaço para o diálogo com o usuário, por mais reduzido que ele seja, abrimos uma porta para que a "vida lá fora" entre na dinâmica do trabalho do serviço de saúde 5 .

E compete aos trabalhadores de saúde, conhecer a história de vida dos usuários, a fim de favorecer escolhas e caminhos a serem seguidos com comprometimento, segurança e esperança de uma resposta positiva ao seu perfil atual ${ }^{15}$. A 
agilidade do acolhimento se dá pelo fato da equipe fazer a escuta. Como todos serão ouvidos de acordo com a sua necessidade, melhora o fluxo dos usuários ${ }^{11}$. Corroborando com a literatura, observou-se que a escuta realizada pelos profissionais de saúde produziu mais agilidade e precisão quanto aos procedimentos terapêuticos, dando espaço ao protagonismo dos sujeitos emergirem no processo de tratamento fonoaudiológico. Além disso, as informações quanto ao funcionamento do serviço e esclarecimentos sobre o próprio fazer da Fonoaudiologia possibilitaram ao usuário o conhecimento de alguns aspectos por vezes idealizados pelo mesmo como, por exemplo, acreditar que o tratamento depende apenas do profissional de saúde. Explica-se também à população a demanda presente no serviço, assim como são expostas as iniciativas realizadas pelos profissionais devido a esta problemática.

Faz-se importante relatar que a proposta do acolhimento também foi observar o indivíduo como um ser único e indivisível, uma vez que a divisão do indivíduo em "pedaços" contribui para dificultar a visão do todo, sendo difícil lidar e/ou compreender as influências de aspectos psicológicos, culturais e subjetivos. No entanto, por mais que os profissionais estejam interessados em ampliar sua visão, torna-se difícil, pois a própria formação leva a raciocinar de maneira a encontrar uma doença, para a qual existe um medicamento a ser prescrito ou uma intervenção a ser realizada, reforçando a influência da dimensão biológica no processo saúde-doença ${ }^{16}$.

Outro aspecto do acolhimento foi a utilização do atendimento em grupo, sendo que o mesmo foi fundamental, visto que o ser humano é um ser instintivamente social, o grupo pode trazer benefícios emocionais e sociais e o acolhimento coletivo pode possuir uma função terapêutica, pela relação estabelecida entre os participantes na discussão de seus problemas, auxiliando o indivíduo em suas relações pessoais e interpessoais, criando situações de diálogo, enfrentamento das dificuldades, funcionando como uma troca de experiências, as quais possam auxiliar na sua reabilitação e/ou na convivência com os demais, do mesmo modo que são consideradas as suas necessidades individuais ${ }^{17,18}$.

Assim sendo, a vida não é o que se passa apenas em cada um dos sujeitos, mas principalmente o que se passa entre os sujeitos, nos vínculos que constroem e que os constroem como potência de afetar e ser afetado. Todavia, os processos de "anestesia" de nossa escuta e de produção de indiferença diante do outro, em relação às suas necessidades e diferenças, têm-nos produzido a enganosa sensação de salvaguarda, de proteção do sofrimento. E esses processos nos mergulham no isolamento, entorpecem nossa sensibilidade e enfraquecem os laços coletivos mediante os quais se nutrem as forças de invenção e de resistência que constroem nossa própria humanidade ${ }^{1}$.

Quanto ao interesse que os profissionais da equipe multiprofissional tiveram em conhecer e estudar a Política Nacional do Humaniza SUS, é importante referir que a formação hospitalocêntrica e biologicista na saúde não vem preparando profissionais com a competência de criar espaços públicos de negociação, de trabalhar em equipe ou de reconhecer, respeitar e integrar o outro. A defesa da vida e do SUS passa por mudanças na formação destes profissionais ${ }^{11}$. Além da reformulação na formação acadêmica ressalta-se ainda a influência dos fatores políticos, econômicos, sociais e históricos que conduzem as ações em saúde tanto dos usuários como dos trabalhadores da saúde. Por isso, a instituição, os profissionais e os usuários precisam estar envolvidos com essa temática para superar os problemas que são inúmeros e de ordem estrutural ${ }^{19}$.

Desta forma, para a conquista de um serviço voltado para as necessidades da população é preciso a colaboração de todos os envolvidos nesse processo, para que, juntos, dividindo saberes, responsabilidades e compromisso, possam estabelecer uma nova prática que convida o repensar ético-político do cotidiano e fortalece a prática do diálogo como forma de apoiar e estimular a criatividade e a singularidade presentes no cotidiano dos serviços de saúde ${ }^{20}$.

\section{CONCLUSÃO}

Os resultados permitiram-nos concluir que implantar o acolhimento na porta de entrada ao serviço de Fonoaudiologia é uma forma de agilizar o acesso da população, assim como escutar implica em respeitar as demandas do usuário.

A experiência do olhar multiprofissional nestes momentos possibilitou o compartilhamento de saberes e angústias e o próprio conhecimento do trabalho das diferentes áreas da saúde, lembrando que, acima de tudo, os profissionais da equipe são profissionais de saúde. Outro fator a ser considerado foi a importância de haver um acolhimento entre os profissionais da equipe, o que não é tarefa fácil tendo em vista a formação clínica das diferentes especialidades.

O estabelecimento de redes apresentou-se como uma dificuldade na proposta de acolhimento no SUS, pois pela significativa demanda e o acúmulo de funções dos profissionais, os mesmos 
não conseguem se responsabilizar de forma integral pelos usuários, assim como falta adesão dos diferentes serviços para que a rede se torne efetiva.

Por fim, é necessário dar continuidade ao estudo de materiais disponibilizados pelo Ministério da Saúde, assim como conhecer as experiências do "SUS que dá certo", permitindo desta forma multiplicar esta experiência com a intenção de re-elaborar e enriquecer o fazer, pois a melhor forma de descobrir o caminho é trilhá-lo.

\begin{abstract}
Background: for the embracement practice in health services to occur it is necessary to develop ways to receive the population, respecting the different modes of how the users seek help. In this way, this assignment is an experience report that had as objective to expedite the access to service of speech therapy, as well as to listen and to give more appropriate answers to the users. Procedures: as a way to structure the access, one accomplished the implementation of embracement's practice in the incoming door at the services of speech therapy at Toledo's city in Parana. For the embracement practice's achievement we established two instances, the first one referred to the waiting list of users which needed to be known and in the second moment the new users choose between days and fixed schedule, as a manner of incoming in services of speech therapy. In some of these meetings there was also the participation of nurses, psychologists, social workers. Results: there was offered to the users a better access to the speech therapy treatment, an enrichment in the professional/user relationship was offered, for adequacy of therapy procedures and explanations about the service functioning. Conclusion: implementing embracement practice at the incoming door for the services of speech therapy, expedited the access for the population, as well as listening also implies in respecting the user's demands.
\end{abstract}

KEYWORDS: User Embracement; Health Services Accessibility; Speech, Language and Hearing Sciences

\section{REFERÊNCIAS}

1. Brasil. Ministério da Saúde. Secretaria de Atenção à Saúde. Núcleo Técnico da Política Nacional de Humanização. Acolhimento nas práticas de produção de saúde. Série B. Textos Básicos de Saúde. 2. ed. Brasília: Editora do Ministério da Saúde, 2006. 44 p.

2. BRASIL. Ministério da Saúde. Secretaria de Atenção à Saúde. Núcleo Técnico da Política Nacional de Humanização. Clínica ampliada, equipe de referência e projeto terapêutico singular. Série B. Textos Básicos de Saúde. 2. ed. Brasília: Editora do Ministério da Saúde, 2007. 60 p.

3. Merhy EE. Em busca do tempo perdido: a micropolítica do trabalho vivo em saúde. In: Merhy EE, Onocko RT. Agir em saúde: um desafio para o público. 2. ed. São Paulo: Hucitec, 2002. p. 71-112. 4. Almeida EC, Furtado LM. Acolhimento em Saúde Pública: A contribuição do fonoaudiólogo. Rev. Ciênc. Méd. 2006, maio/jun; 15(3): 249-56.

5. Takemoto MLS, Silva EM. Acolhimento e transformações no processo de trabalho de enfermagem em unidades básicas de saúde de Campinas, São Paulo, Brasil. Cad. Saúde Pública. 2007, fev; 23(2): 331-40.

6. Malta DC, Ferreira LM, Reis AT, Merhy EE. Mudando o processo de trabalho na rede pública: alguns resultados da experiência de Belo Horizonte. Saúde Debate. 2000, 1(24): 21-34.

7. Mendes VLF. Grupos, instituições e processos grupais. In: Tomé MC. (org.). Dialogando com o coletivo: Dimensões da saúde em fonoaudiologia. São Paulo: Santos, 2009. p. 107-20.

8. Brasil. Ministério da Saúde. Secretaria de Atenção à Saúde. Núcleo Técnico da Política Nacional de Humanização. HumanizaSUS: Documento base para gestores e trabalhadores do SUS. Série B. Textos Básicos de Saúde. 4. ed. - Brasília : Editora do Ministério da Saúde, 2008. 72 p.

9. Donabedian A. Aspects of medical care administration. Boston: Harvard University Press; 1973. p. $130-40$.

10. Cruz SV, Ribeiro LD, Cabral LHA, Sampaio RF. O olhar do usuário sobre o acolhimento em um 
serviço de reabilitação. Acta Fisiatr. 2010; 17(3): 122-5.

11. Cavalcante Filho JB, Silva VEM, Burg CR, Bezerra GL. Acolhimento coletivo: um desafio instituinte de novas formas de produzir o cuidado. Interface - Comunic., Saúde, Educ. 2009, out./dez., 13(31):315-28.

12. Beck CLC, Lisbôa RL, Tavares JP, Silva RM, Prestes FC. Humanização da assistência de enfermagem: percepção de enfermeiros nos serviços de saúde de um município. Rev Gaúcha Enferm. 2009, mar., 30(1):54-61.

13. Tesser CD, Poli Neto $P$, Campos GWS. Acolhimento e (des)medicalização social: um desafio para as equipes de saúde da família. Cienc. Saude Colet. 2010, Nov., 15(supl.3):3615-24.

14. Almeida MCP, Mishima SM, Silva EM, Mello DF. O trabalho de enfermagem e sua articulação com o processo de trabalho em saúde coletiva rede básica de saúde. In: Almeida MCP de, Rocha SMM. (orgs.). O trabalho de enfermagem. São Paulo: Cortez, 1997. p. 61-112.

15. Pereira AD, Freitas HMB, Ferreira CLL, Marchiori MRCT, Souza MHT, Backes DS. Atentando para as singularidades humanas na atenção à saúde por meio do diálogo e acolhimento. Rev Gaúcha Enferm. 2010, mar, 31(1):55-61.

16. Barros JA. Pensando o processo saúde/doença, a que responde o modelo biomédico? Saude Soc. 2002, jan./jul. 11(1): 67-84.

17. Souza APR, Crestani AH, Vieira CR, Machado FCM, Pereira LL. O grupo na fonoaudiologia: origens clínicas e na saúde coletiva. Rev CEFAC 2011, fev., 13(1): 140-51.

18. Botti N, Andrade W. A saúde mental na atenção básica - articulação entre os princípios do SUS e da reforma psiquiátrica. Cogitare Enferm. 2008, jul/set; 13(3):387-94.

19. Medeiros FA, Araujo-Souza GC de, AlbuquerqueBarbosa AA, Clara-Costa IC. Acolhimento em uma Unidade Básica de Saúde: a satisfação do usuário em foco. Rev. Salud Pública. 2010, jun., 12(3): 402-13.

20. Coelho MO, Jorge MSB, Araújo ME. O acesso por meio do acolhimento na atenção básica à saúde. Rev. Baiana Saúde Pública 2009, jul./set., 33(3): 440-52.
http://dx.doi.org/10.1590/S1516-18462012005000007

RECEBIDO EM: 27/10/2010

ACEITO EM: 28/07/2011

Endereço para correspondência:

Juliana Cunha da Costa

Rua Presidente José Linhares, 423

Jardim Filadélfia - Toledo - PR

CEP: 85902-350

E-mail: jccfono@yahoo.com.br 